\title{
Exposición infantil al plomo en sitios contaminados
}

\author{
Rogelio Flores-Ramírez, M en C,(1) Edna Rico-Escobar, D en C, , $^{(2)}$ Jorge E Núñez-Monreal, M en C,(3) \\ Edelmira García-Nieto, D en C, (4) Leticia Carrizales, QFB, (I) César llizaliturri-Hernández, D en C, (I) \\ Fernando Díaz-Barriga, D en C. ${ }^{(1)}$
}

\section{Flores-Ramírez R, Rico-Escobar E, Núñez-Monreal JE, García-Nieto E, Carrizales L, Ilizaliturri-Hernández C, Díaz-Barriga F. Exposición infantil al plomo en sitios contaminados. Salud Publica Mex 20I2;54:383-392.}

\begin{abstract}
Resumen
Objetivo. Determinar el grado de la exposición infantil al plomo en diversos tipos de sitios contaminados. Material y métodos. El estudio se realizó de junio 2008 a diciembre 2009 en cuatro sitios de México: metalúrgica de Ávalos, Chihuahua.; metalúrgica de Morales, San Luis Potosí (SLP); zona alfarera en La Trinidad, Tlaxcala, y sitio minero en Cedral, SLP. Se cuantificó plomo en polvo y se realizó un biomonitoreo humano en niños de la comunidad. Resultados. Los valores obtenidos de plomo en polvo exterior superaron el límite establecido de $400 \mathrm{mg} / \mathrm{kg}$ para suelos residenciales en un intervalo de valores para los cuatro sitios de 62 a 5187 $\mathrm{mg} / \mathrm{kg}$. En cuanto al monitoreo biológico, todas las poblaciones presentaron valores extremos, desde los $22 \mu \mathrm{g} / \mathrm{dL}$ en Cedral, $31 \mu g / d L$ en Morales, y $32 \mu g / d L$ en Ávalos, hasta los $52 \mu g /$ $\mathrm{dL}$ en La Trinidad. Es importante señalar que encontramos una correlación positiva y significativa entre los valores de plomo en polvo y plomo en sangre en todos los sitios de estudio $(p<0.001)$. Conclusión. Estos sitios son un ejemplo de los riesgos en salud relacionados con la exposición a plomo en México; por consiguiente, se requiere de un programa nacional de salud pública dirigido a reducir la exposición a este metal en poblaciones vulnerables.
\end{abstract}

Palabras clave: plomo; sitios contaminados; monitoreo biológico; salud infantil; México
Flores-Ramírez R, Rico-Escobar E, Núñez-Monreal JE, García-Nieto E, Carrizales L, Ilizaliturri-Hernández C, Díaz-Barriga F.

Children exposure to lead in contaminated sites. Salud Publica Mex 20I2;54:383-392.

\begin{abstract}
Objective. To assess the exposure to lead in children living in various types of contaminated sites. Materials and methods. The study was conducted from June 2008 to December 2009 at four sites in Mexico: Avalos metallurgical, Chihuahua; Morales metallurgical, San Luis Potosí (SLP); Trinidad pottery area, Tlaxcala and Cedral mine site, SLP. These sites contain different sources of lead. The metal levels were quantified in outdoor dust and in peripheral blood of children. Results. Lead dust concentrations exceed the National Guidelines for residential soils $(400 \mathrm{mg} / \mathrm{kg})$ in a range of values for the four sites from 62 to $5187 \mathrm{mg} / \mathrm{kg}$. Regarding biological monitoring, the studied children showed maximum lead blood levels of $22 \mu \mathrm{g} / \mathrm{dL}$ in Cedral, $31 \mu \mathrm{g} / \mathrm{dL}$ in Morales, $32 \mu \mathrm{g} / \mathrm{dL}$ in Avalos, and $52 \mu \mathrm{g} / \mathrm{dL}$ in Trinidad. It is important to mention that in all the studied sites, a significative positive correlation was found between blood lead levels and the lead concentrations in dust. Conclusion. These sites are an example of the health risks related to lead exposure in Mexico; therefore, there is an urgent need for a national public health program aimed at reducing lead exposure in vulnerable populations.
\end{abstract}

Key words: lead; hazardous sites; biological monitoring; children's environmental health; Mexico

(I) Centro de Investigación Aplicada en Ambiente y Salud, CIACYT-Medicina, Universidad Autónoma de San Luis Potosí. México.

(2) Centro de Investigaciones Biomédicas, Universidad Autónoma de Ciudad Juárez. Baja California, México.

(3) Facultad de Química, Universidad Autónoma de Zacatecas. México.

(4) Centro de Investigación en Genética y Ambiente, Universidad Autónoma de Tlaxcala. México. 
$E^{1}$ plomo es un elemento de reconocida capacidad tóxica que afecta a toda la población. ${ }^{1}$ Sin embargo, los niños son más susceptibles dado que, en relación con su masa, inhalan más aire e ingieren mayores cantidades de suelo que los adultos; pero además, la interacción mano-boca, tan común durante la infancia, facilita la ingesta de polvo. ${ }^{2}$ Por si estos datos no fueran suficientes, es necesario considerar que la absorción gastrointestinal del plomo en niños es hasta cinco veces superior que en el adulto y la distribución a órganos extra-óseos (incluyendo el sistema nervioso central) también es mayor. ${ }^{1,3}$

Aunque actualmente las alteraciones neurocognitivas destacan como el efecto de mayor preocupación para la población infantil expuesta al metal, también se han demostrado efectos auditivos, cardiovasculares, nefrológicos y hematológicos entre niños expuestos a plomo. ${ }^{1-5}$ La definición de las concentraciones a las cuales este tóxico afecta los diferentes órganos y sistemas del niño todavía requiere de mayores investigaciones, pero existe evidencia que la toxicidad del plomo inicia a concentraciones corporales bajas. Por ejemplo, ha sido demostrada la capacidad del metal para generar cambios neurocognitivos a concentraciones alrededor de $5.0 \mu \mathrm{g} / \mathrm{dL}$ del tóxico en sangre. ${ }^{6-9}$

Con estos antecedentes, se entienden los esfuerzos por controlar la exposición al plomo; así, en la gran mayoría de los países, el metal ha sido eliminado de las gasolinas. Sin embargo, ante la nueva evidencia científica sobre su toxicidad a bajas concentraciones, mayores esfuerzos se realizan para evitar su presencia en pinturas, ${ }^{10}$ alimentos ${ }^{11}$ y aire. ${ }^{12}$ México no escapa a estos esfuerzos, y ya a partir de 1986 y con la participación de diversos científicos, ${ }^{13}$ fue posible gestionar la producción de gasolinas libres de plomo. No obstante este logro, ahora se requiere controlar otras fuentes, dado que diversos estudios en distintas entidades del país han mostrado la existencia de niños expuestos al plomo y en diversos casos la exposición ha sido relacionada con efectos en la salud. ${ }^{9,13-23}$

Entre las fuentes de plomo que habría que controlar, además del barro vidriado, están los sitios contaminados donde el tóxico puede encontrarse en aire, polvo, suelo, agua y alimentos, y que afectan los diversos ambientes infantiles (casa, escuela y áreas de recreación). Los sitios contaminados con plomo donde los niños juegan, estudian y viven implican un riesgo para la salud infantil.

En este contexto, el objetivo del presente trabajo fue determinar el grado de exposición infantil al plomo en diversos tipos de sitios contaminados, con el fin de definir cuáles son los escenarios que podrían requerir mayores estudios para reducir la exposición infantil a este metal.

\section{Material y métodos Descripción de los sitios de estudio}

Ávalos, Chihuahua. La comunidad de Ávalos se encuentra en la zona sur de la ciudad de Chihuahua, aproximadamente a unos 600 metros de una antigua fundición de plomo. La fundición de Ávalos inició operaciones en 1908 y en los últimos años de operación produjo alrededor de 250 toneladas diarias de este metal. En 1993 inició el plan de cierre que concluyó en 1998. Una evaluación preliminar en el sitio demostró exposición a plomo. ${ }^{24}$ Actualmente, el sitio se encuentra bajo restauración ambiental (control del material contaminado).

Morales, San Luis Potosí. Este sector de la ciudad de San Luis Potosí es vecino de una zona metalúrgica compuesta por una fundición de cobre y una electrolítica de zinc. Al momento del presente estudio, en la fundición de cobre, además de cobre y arsénico, se producían concentrados de plomo. La empresa inició actividades durante 1890 e, inclusive, de 1926 a 1959 operó una fundición de plomo. La exposición infantil a plomo en Morales ha sido previamente reportada. ${ }^{25}$ Recientemente, la planta de cobre cerró, pero la electrolítica de zinc mantiene funciones.

Cedral, San Luis Potosí. La cabecera municipal de Cedral se ubica en la zona del altiplano del estado de San Luis Potosí. Este sitio formó parte de un sistema industrial minero-metalúrgico que ha funcionado desde 1615 y hasta la actualidad en un área denominada "región minera de la sierra de Catorce" que corresponde a los municipios de Vanegas, Cedral, Catorce, Villa de La Paz y Matehuala. En la comunidad se ubicaron haciendas de beneficio que procesaban el mineral que procedía de las minas de Real de Catorce, utilizando el método de patio (amalgamación con mercurio). Como desechos de este método, se generaron los jales de Jesús María y el Caballo, localizados dentro de la zona urbana de Cedral. El presente es el primer estudio de exposición infantil efectuado en la localidad. El sitio fue seleccionado debido a que estudios preliminares mostraron un alto contenido de plomo en los jales mineros.

Trinidad Tenexyecac, Tlaxcala. La Trinidad Tenexyecac se ubica en la parte centro-poniente del estado de Tlaxcala, México. Su actividad histórica y tradicional desde 1872 es la elaboración de artesanías, principalmente cazuelas de barro vidriado, en donde utiliza la greta (óxido de plomo) como barniz. El uso de estos recipientes refleja la tradición alimentaria de Tlaxcala de preparar en ellos alimentos tales como mole, arroz y frijol. Este es el primer estudio de exposición infantil a plomo en la comunidad alfarera de La Trinidad. 


\section{Evaluación ambiental de plomo en polvo}

Para la selección de las muestras de polvo se realizó un muestreo dirigido de las zonas de estudio entre junio de 2008 y diciembre de 2009. Las muestras se colectaron en puntos similares a la residencia de los niños (figura 1). Se incluyeron puntos de exposición como banquetas, exteriores de casas, áreas recreativas, patios y zonas escolares. Para la obtención de polvo se delimitó un área de $1 \mathrm{~m}^{2}$ y con ayuda de una brocha se recolectó el material, que fue almacenado en bolsas de polietileno. Posteriormente, la muestra se tamizó en una criba de acero inoxidable y se recuperaron partículas menores a $250 \mu \mathrm{m}$. Se aseguró que los materiales de recolección estuvieran libres de plomo. Se obtuvo una cantidad total de 36 muestras de polvo en Ávalos, 35 en Morales, 49 en Cedral y 11 en La Trinidad. En todos los casos se registraron las coordenadas geográficas de los puntos de muestreo empleando un GPS. Las muestras fueron digeridas de acuerdo con el método EPA $3051^{26}$ y siguiendo las recomendaciones de la norma mexica- na NOM-147-SEMARNAT/SSA1-2004, ${ }^{27}$ se determinó la concentración de plomo en polvo por la técnica de espectrometría de absorción atómica por flama.

\section{Evaluación de la exposición}

Se cuantificaron las concentraciones de plomo en sangre en niños de preescolar y primaria, seleccionando voluntarios en zonas de alto riesgo, para lo cual se trabajó en áreas cercanas a la fuente de exposición. Para el caso de Morales, Cedral y Ávalos se tiene identificada la fuente de exposición, no así para La Trinidad debido a que los talleres alfareros se encuentran distribuidos alrededor de la comunidad. En la figura 1 se muestra la distribución de la población en estudio. Tomando en cuenta que el objetivo era evaluar la magnitud y las fuentes del problema, tal y como ha sido recomendado en la literatura, ${ }^{28}$ se emplearon los siguientes métodos de muestreo: a) muestreo objetivo de los grupos de alto riesgo; y b) muestreo de conveniencia (de voluntarios), utilizando la cuantificación de plomo en sangre como indicador de riesgo. En ningún

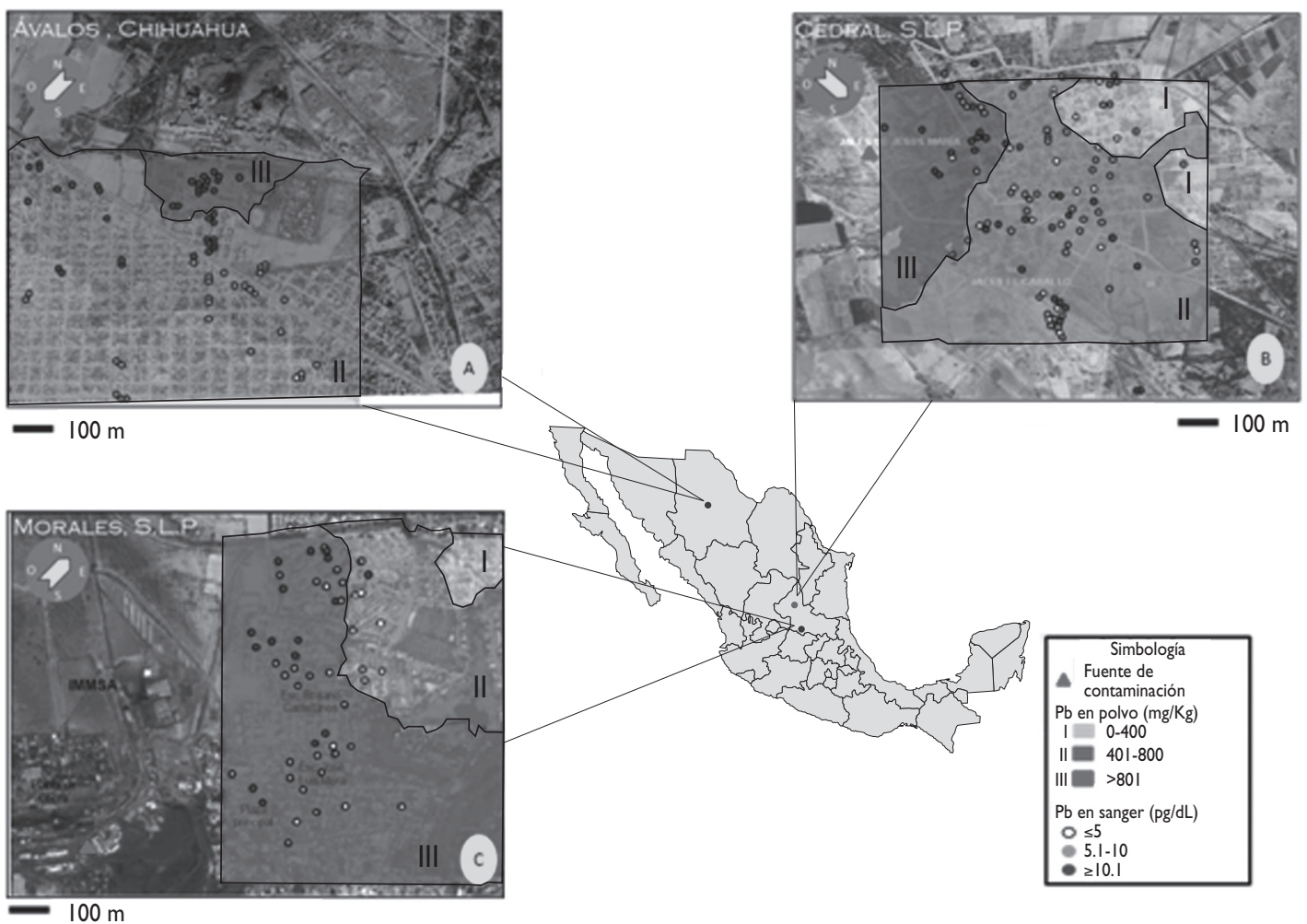

Nota: Distribución de la concentración de plomo en polvo de los sitios de Ávalos Chihuahua (A), Cedral SLP (B) y Morales, SLP. (C) y la concentración de plomo en sangre de niños. Los puntos representan a cada uno de los niños muestreados y la concentración de plomo en sangre con valores $\leq 5,5$. I- I0 $y \geq 10 \mu \mathrm{g} / \mathrm{dL}$. Las muestras fueron colectadas en el periodo de junio de 2008 y diciembre de 2009

Figura I. Mapas de isoconcetración de plomo en polvo y distribución de los niños muestreados en los sitios CONTAMINADOS POR ESTE METAL 
momento el presente trabajo tuvo como finalidad que la muestra fuese representativa de la comunidad pues sólo se buscó definir el nivel de exposición para tomar medidas de intervención. ${ }^{28}$

Los criterios de inclusión que se tomaron en cuenta para la participación del niño en el estudio fueron: 1) consentimiento informado, voluntario y firmado por los padres de cada niño para realizar la toma de la muestra, 2) tiempo de residencia mínimo de 2 años, y 3) edad entre 4 y 9 años. Se aplicó un cuestionario a los padres de familia para identificar el sitio exacto de residencia y ubicarlos en el mapa de isoconcentración, las probables rutas y fuentes de exposición, hábitos del niño, alimentación y estado socieconómico, entre otros.

En total se lograron 67 muestras en Ávalos, 58 en Morales, 119 en Cedral, y 72 en La Trinidad. Las muestras de sangre fueron obtenidas por punción venosa de la vena antecubital con tubos Vacutainer al vacío, libres de plomo y con EDTA como anticoagulante. Posteriormente se almacenaron a $4^{\circ} \mathrm{C}$ hasta su análisis.

Se determinó y cuantificó la concentración del metal en sangre mediante un espectrofotómetro de absorción atómica con horno de grafito, y se utilizó un modificador de matriz (difosfato de amonio-tritón X-100 en $\mathrm{HNO}_{3}$ al $2 \%$ ) siguiendo el método de Subramanian. ${ }^{29} \mathrm{El}$ trabajo contó con la aprobación del comité de bioética de la Facultad de Medicina de la Universidad Autónoma de San Luis Potosí.

\section{Control de calidad de los análisis ambiental y biológico}

Las cuantificaciones de las muestras se realizaron en el laboratorio de toxicología ambiental de la Facultad de Medicina de la Universidad Autónoma de San Luis Potosí. Previo al análisis químico de las muestras ambientales y biológicas, se realizó la validación de los métodos analíticos, estableciendo parámetros de exactitud, linealidad, repetibilidad, límite de detección(LOD) y cuantificación (LOC) (cuadro I). El LOD y el LOC se calcularon con los resultados obtenidos del triplicado de la curva de calibración de 1-100 mg/ $\mathrm{kg}$ (polvo) y 1-100 $\mu \mathrm{g} / \mathrm{L}$ (sangre) y fueron determinados mediante una curva lineal a bajas concentraciones, sumándole el límite de confianza al 95\%. ${ }^{30-31}$ La linealidad expresada por el coeficiente de correlación (r) y la sensibilidad determinada por la pendiente de la curva $(\mathrm{m})$ se obtuvieron del promedio de once curvas trabajadas durante cinco días. La precisión de la metodología se obtuvo como repetibilidad al evaluar la curva de calibración el mismo día por triplicado. La exactitud se obtuvo a partir de un control de calidad interno que analiza estándares de referencia

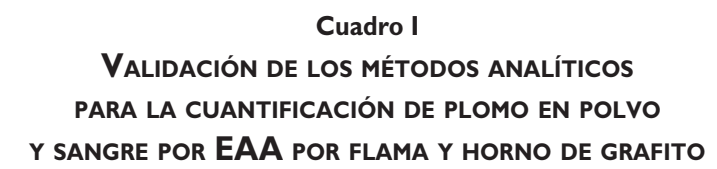

\begin{tabular}{lll} 
Parámetro & Pb polvo & Pb sangre \\
Sensibilidad (m) & 0.008 & 0.0045 \\
\hline Intercepto (b) & 0.002 & 0.002 \\
\hline Coeficiente de determinación ( 2$)$ & 0.9996 & 0.999 \\
\hline Repetibilidad (\%RDS) & 7.6 & 5 \\
\hline Exactitud (\%) & $102.3-111.7$ & $95-105$ \\
\hline LOD & $0.15 \mathrm{mg} / \mathrm{kg}$ & $0.1 \mu \mathrm{gg} /$ \\
\hline LOC & $0.67 \mathrm{mg} / \mathrm{kg}$ & $0.7 \mu g / /$ \\
EAA $=$ Espectrofotómetro de absorción atómica & \\
LOD $=$ Límite de detección & & \\
LOC $=$ Límite de cuantificación &
\end{tabular}

certificado por el Instituto Nacional de Estándares y Tecnología y el Centro de Control de Enfermedades de los Estados Unidos (NIST y CDC, por sus siglas en inglés). Para polvo se empleó el SRM 2710 y sangre CDC.WS2 H (cuadro I). El análisis de cada SRM y CDC se hizo por cada lote de 50 muestras. En todos los casos se obtuvieron recuperaciones aceptables.

\section{Análisis estadístico}

Se realizó un análisis de normalidad mediante la prueba Kolmogorov-Smirnov para los datos de plomo en polvo y sangre. Las variables no presentaron una distribución normal, por lo tanto, el análisis estadístico se realizó con la mediana y como medida de dispersión el rango intercuartílico; con fines comparativos se obtuvo la media geométrica de cada sitio. Se analizaron los datos de plomo en polvo y sangre por sitio, mediante la prueba de contraste de Kruskal-Wallis, seguido de una comparación múltiple de Dunn (post-hoc) a un nivel de significancia de $p<0.05$. Aunado a lo anterior, se efectuó un análisis de correlación de Spearman (r) para las variables de plomo en polvo y sangre en cada uno de los sitios de estudio. Los datos fueron analizados por medio del paquete estadístico GraphPad Prism 5.

\section{Resultados}

En el cuadro II se presentan las concentraciones de plomo en polvo, las cuales fueron altas en los cuatro sitios de estudio. Dado que no existen valores de referencia para este metal en polvo, se realizó una comparación 
de los niveles registrados en el área de estudio contra los valores de referencia de plomo en suelo que señala la NOM-147-SEMARNAT/SSA1-2004. Es importante hacer notar que de existir valores de referencia de plomo en polvo, éstos deberían ser de magnitud menor a los valores de suelo, debido a que las partículas de polvo presentan mayor biodisponibilidad y capacidad de entrar al organismo tanto por ingesta como por inhalación. En el cuadro II se observa que todos los sitios estudiados tuvieron valores máximos de 7 a 13 veces por arriba de la normativa para plomo en suelos residenciales $(400 \mathrm{mg} / \mathrm{kg})$. Asimismo, se indica el alto porcentaje de muestras que presentaron valores por arriba de esta guía ambiental, desde 34\% de Cedral hasta 100\% para la comunidad de La Trinidad. Al realizar la comparación entre los sitios, se encontraron diferencias significativas entre Ávalos, La Trinidad y Morales con respecto a Cedral, que fue el sitio que presentó los niveles más bajos $(p<0.0001, p<0.001, \mathrm{y} p<0.05$ respectivamente).

Al tomar en cuenta el alto contenido de plomo en las muestras de polvo exterior, se evaluó el nivel de plomo en sangre en los niños de los cuatro sitios. Los resultados se indican en el cuadro III. Las medias geométricas (Media G) en Ávalos y en La Trinidad superaron la norma establecida de $10 \mu \mathrm{g} / \mathrm{dL} .^{32}$ Llama la atención, además, que La Trinidad superó esta guía desde su percentil 25. Ahora bien, nótese que en todos los sitios hubo niños con valores máximos en extremo altos, desde los $22 \mu \mathrm{g} / \mathrm{dL}$ en Cedral, $31 \mu \mathrm{g} / \mathrm{dL}$ en Morales, y $32 \mu \mathrm{g} / \mathrm{dL}$ en Ávalos, hasta los $52 \mu \mathrm{g} / \mathrm{dL}$ en La Trinidad. Asimismo, se observa el alto porcentaje de niños con valores por arriba del límite propuesto de $5.0 \mu \mathrm{g} / \mathrm{dL}$ y adicionalmente, en La

Cuadro II

Concentración de plomo en polvo en sitios contaminados de México, 2008 - 2009

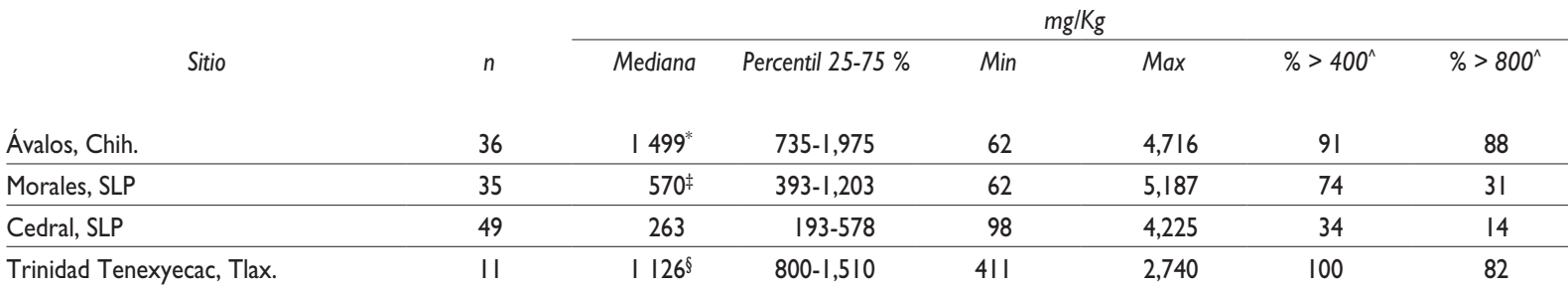

Entre junio de 2008 y diciembre de 2009, se colectaron muestras de polvo en cada uno de los sitios señalados en el cuadro. Los sitios tienen como característica diferentes fuentes de emisión de este metal. Los valores de plomo en polvo tuvieron diferencias estadísticamente significativas:

* Ávalos vs Cedral $(p<0.0001)$

$\ddagger$ Morales vs Cedral $(p<0.05)$

$\S$ Trinidad vs Cedral $(p<0.00 \mathrm{I})$. Los resultados están presentados como mediana con su percentil 25 y $75 \%$, concentración mínima (Min) y máxima (Max) encontradas en las muestras. En las últimas dos columnas el porcentaje de muestras por arriba de los valores de referencia. ^NOM-I47-SEMARNAT/SSAI2004. Límite Máximo Permisible por tipo de uso de suelo agrícola/residencial/comercial: $400 \mathrm{mg} / \mathrm{Kg}$; industrial: $800 \mathrm{mg} / \mathrm{Kg}$

\section{Cuadro III \\ Concentración de plomo en sangre de niños Que viven en sitios contaminados de México, 2008-2009}

\begin{tabular}{|c|c|c|c|c|c|c|c|c|}
\hline \multirow[b]{2}{*}{ Sitio } & \multirow[b]{2}{*}{$n$} & \multicolumn{7}{|c|}{$\mu \mathrm{g} / \mathrm{dL}$} \\
\hline & & Mediana & Percentil $25-75 \%$ & Media G & Min-Max & $\%>5$ & $\%>10$ & $\%>20$ \\
\hline Ávalos, Chih. & 67 & $10.4^{\ddagger}$ & $8.8-13.4$ & 11.3 & $4.7-32.1$ & 98 & 57 & 13 \\
\hline Morales, SLP & 58 & 7.1 *., $^{-1}$ & $4.9-10.5$ & 7.1 & $||-3 \mid .0$. & 74 & 22 & 5 \\
\hline Cedral, SLP & 119 & $5.8^{*, \neq}$ & 4.4-8.7 & 6.1 & $0.4-22.0$ & 65 & 18 & 1 \\
\hline Trinidad Tenexyecac, Tlax. & 72 & $19.2^{*}$ & $14.8-25.0$ & 19.4 & $6.2-52.3$ & 100 & 93 & 47 \\
\hline
\end{tabular}

Entre junio de 2008 y diciembre de 2009, se colectaron muestras de sangre en niños de 4 a 9 años que nacieron y viven en cada uno de los sitios señalados en el cuadro. Los sitios tienen como característica diferentes fuentes de emisión de este metal. Las muestras fueron estadísticamente significativas con:

* Ávalos

‡ Trinidad, en ambos casos $p<0.000$ I. Los resultados son presentados como mediana, percentil 25 y $75 \%$, media geométrica (Media G), concentración mínima (Min) y máxima (Max) encontrados en las muestras. En las tres últimas columnas se presenta el porcentaje de niños por arriba de los respectivos valores de referencia 
Trinidad se encontró un elevado porcentaje de niños con valores por arriba de $20.0 \mu \mathrm{g} / \mathrm{dL}$. La comparación entre los sitios muestra que La Trinidad y Ávalos presentan diferencias significativas con respecto al resto de los sitios $(p<0.0001)$.

El riesgo por exposición a plomo se hace evidente cuando los valores de plomo en sangre de los niños se compartamentalizan de acuerdo con los valores fijados por la normativa mexicana (cuadro IV). ${ }^{32}$ En tres sitios (Ávalos, Morales y La Trinidad) tenemos niños hasta en el sector III y IV, e inclusive, La Trinidad presenta niños en el sector $\mathrm{V}$ (máximo nivel de la normativa). En la figura 1 se presenta la distribución de los niveles de plomo en polvo, la localización de la vivienda de los niños y sus valores sanguíneos de plomo. Puede advertirse que la dirección de los vientos predominantes proporciona un factor favorable para la distribución del plomo y con ello se incrementa el riesgo a la exposición. Finalmente, se encontró que existe una correlación significativa ( $p<$ $0.001)$ entre los valores de plomo en sangre y plomo en polvo $(\mathrm{r}=0.9031,0.9581,0.5816$ y 0.8244 para Ávalos, Morales, Cedral y La Trinidad, respectivamente).

\section{Discusión}

El presente estudio indica que en los sitios analizados existe una alta proporción de niños con niveles de plomo en sangre por arriba de los límites recomendados por la normativa mexicana. Asimismo, el trabajo demuestra que una de las rutas de exposición sería el polvo que puede ingresar al organismo por inhalación o ingesta. No obstante, otras rutas de exposición son factibles, tales como la contaminación de alimentos, el uso de barro vidriado, la ingesta de suelo, la exposición a pinturas, entre otras. Si bien estas rutas no fueron evaluadas en el presente estudio, tal hecho no se contrapone al prin- cipal resultado, esto es, que en sitios contaminados con plomo, el porcentaje de niños con valores del metal en sangre por arriba de los valores recomendados es alto.

Los resultados obtenidos en la zona minera de Cedral, si bien son altos, son menores a los que se presentan en otras zonas mineras de México o del mundo. Por ejemplo, en Cedral 18\% de los niños estudiados tuvo registros de plomo en sangre superiores a los $10 \mu \mathrm{g} /$ dL; en tanto, en niños de la zona minera de Vetagrande en el estado de Zacatecas este porcentaje fue de $45 \%$, 33 y en comunidades del Perú aledañas a jales mineros la guía fue superada por $85 \%$ de los niños estudiados. ${ }^{34} \mathrm{De}$ hecho, en el trabajo realizado en Perú, la concentración promedio de plomo en sangre fue de $15.8 \mu \mathrm{g} / \mathrm{dL}$, contra la media de $6.1 \mu \mathrm{g} / \mathrm{dL}$ registrada en Cedral. Tomando en cuenta que tanto en Vetagrande como en las comunidades peruanas imperan condiciones geográficas similares a Cedral, la diferencia de exposición podría estar dada por la diferente biodisponibilidad del plomo en cada sitio o en la accesibilidad de los niños a los jales mineros. En conclusión, los trabajos aquí referidos y los datos de Cedral apuntan a que la minería de plomo puede ser de alto riesgo para los niños, por lo cual es importante hacer el señalamiento de la abundancia en México de pasivos mineros históricos (de la época colonial) similares a los de Cedral. Dichos pasivos se localizan en San Luis Potosí, Guanajuato, Hidalgo, Zacatecas, Guerrero, etc. De hecho, en el siglo XVI ya existían en nuestro país 237 haciendas de beneficio ${ }^{35} \mathrm{y}$ para el siglo XVIII, y solamente en la zona de Cedral, trabajaban 111 minas y 79 haciendas de beneficio. ${ }^{36}$ Sin embargo, además de los pasivos históricos, la minería activa es también un riesgo. México ocupa el quinto lugar mundial en cuanto a producción de plomo ${ }^{37}$ y dos datos apuntan a que la producción minera mexicana se incrementará: por un lado se planea una fuerte inversión

\section{Cuadro IV \\ Porcentaje de niños de acuerdo con las categorías de la Norma Oficial Mexicana 199-SSAI-2000 SOBRe PLOMO EN SANGRE. MÉXICO 2008-2009}

\begin{tabular}{|c|c|c|c|c|c|c|}
\hline & & \multicolumn{5}{|c|}{ Porcentaje de niños por categoría } \\
\hline Sitio & $n$ & I & II & III & IV & 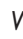 \\
\hline Ávalos, Chih. & 67 & 43 & 34 & 14 & 9 & - \\
\hline Morales, SLP & 58 & 72 & 20 & 4 & 4 & - \\
\hline Cedral, SLP & 119 & 81 & 12 & 7 & - & - \\
\hline Trinidad Tenexyecac, Tlax. & 72 & 7 & 15 & 48 & 23 & 7 \\
\hline
\end{tabular}

Entre junio del 2008 y diciembre del 2009, se colectaron muestras de sangre en niños de 4 a 9 años que nacieron y viven en cada uno de los sitios señalados en el cuadro. Los niños viven en sitios contaminados de México que tienen como característica diferentes fuentes de emisión de este metal. Las categorías I

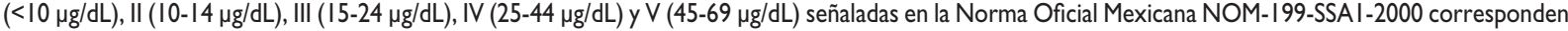
a niveles de plomo en sangre y acciones como criterios para proteger la salud de la población expuesta no ocupacionalmente 
económica y, por otro, actualmente hay 738 proyectos de exploración minera por todo el territorio nacional. ${ }^{37}$ Asimismo, la industria minera ocupa ya el cuarto lugar en cuanto a divisas generadas en México (incluyendo a las remesas). ${ }^{37}$

En cuanto a las metalúrgicas, los resultados en Ávalos y Morales indican que este tipo de empresas también son fuente importante de exposición a plomo. Dicha conclusión se refuerza con estudios efectuados tanto en México ${ }^{22-25,38-40}$ como en otros países. ${ }^{41-43}$ Por ejemplo, trabajos realizados alrededor de una metalúrgica de plomo en la ciudad de Torreón, Coahuila, demostraron que un alto porcentaje de los niños estudiados registraron exposición a plomo y en algunos casos con valores del metal en sangre al doble o triple de lo encontrado en Ávalos y Morales. ${ }^{22,23,38,40}$ Este hecho se explica porque Torreón es una fundición activa con rutas en aire, suelo y polvo, mientras que la fundición de Ávalos al momento del estudio ya se encontraba fuera de operación (la ruta de aire, salvo por partículas de polvo, estaría eliminada). Por su parte, Morales es una fundición de cobre, por lo cual la producción de plomo es menor. Ahora bien, es cierto que aquí se estudiaron solamente grandes fundiciones primarias (Ávalos y Morales), pero también las fundiciones de reciclaje (plomo secundario) son fuentes potenciales de contaminación. Es importante hacer notar que se produce casi tanto plomo primario como secundario o refinado. ${ }^{44}$ Es más, en nuestro país existen al menos 18 recicladoras de plomo y lo grave es que algunos reportes señalan que dichas empresas llegan a emitir hasta 10 veces más plomo al aire que sus contrapartes en Estados Unidos. ${ }^{45}$ Esto es de llamar la atención ya que de Estados Unidos llegan a nuestro país al menos 20 millones de baterías de automóviles para su reciclado. ${ }^{46}$ Un estudio efectuado en China en comunidades con metalúrgicas secundarias de plomo demostró que la población infantil se encuentra altamente expuesta a este metal; el promedio de plomo en sangre entre los niños estudiados fue de $49.6 \mu \mathrm{g} / \mathrm{dL}{ }^{47}$

En cuanto a sitios artesanales, se han reportado al menos 123 comunidades similares a La Trinidad en 20 entidades de nuestro país. ${ }^{48}$ En ellas habría 10000 talleres de alfareros registrados ante el Fondo Nacional para el Fomento de las Artesanías (Fonart) que están en riesgo de padecer una intoxicación por plomo al usar vidrio plomado como esmalte para sus piezas. ${ }^{49}$ Por eso los resultados de Tlaxcala alarman, pero llama más la atención lo poco que han sido estudiadas las comunidades alfareras. ${ }^{50,51}$ En estos trabajos realizados en comunidades alfareras de Jalisco y Oaxaca los resultados de plomo en sangre fueron similares a los obtenidos en el presente trabajo en Tlaxcala. Esto es, un muy alto porcentaje de niños en comunidades alfareras presenta valores de plomo en sangre por arriba de $10 \mu \mathrm{g} / \mathrm{dL}$. La fuente principal en estos casos sería la exposición al polvo, sobre todo en el ambiente de los talleres donde la concentración de plomo es tan alta como en la vecindad a una fundición de plomo (cuadro II).

Ahora bien, los pasivos mineros históricos, las comunidades artesanales o las empresas metalúrgicas no comprenden en conjunto el universo total de fuentes de plomo en México. Por ejemplo, SEMARNAT tiene un listado de 1141 emisores de plomo ${ }^{52}$ y en este listado no se consideran otras potenciales fuentes como los 15000 hornos ladrilleros que operan en nuestro país sin control ambiental algun ${ }^{53}$ o los más de 500 sitios contaminados identificados por el Gobierno Federal. ${ }^{54}$

$\mathrm{Al}$ tomar en cuenta todas las posibles fuentes de emisión de plomo en México, se entiende la alta exposición a este metal encontrada no solamente en el presente estudio sino en otros muchos trabajos realizados en este país. El riesgo, entonces, es evidente y más si consideramos que la toxicidad del plomo es ampliamente reconocida, ${ }^{1,3}$ sobre todo para la población infantil. ${ }^{1,55}$ Por lo tanto, los niños de Cedral, Ávalos, Morales y La Trinidad están en riesgo, y por los datos encontrados, la exposición puede estar asociada con alteraciones neurocognitivas, neuroconductuales, cardiovasculares y de función renal, ${ }^{1,3-9}$ tal y como ocurre en diversos escenarios de México. 9,14-16,19,20

En estos sitios se hace necesaria la instalación inmediata de un programa de salud pública, cuyo objetivo sea reducir la exposición al plomo para así disminuir las consecuencias de los efectos del metal en los diversos órganos y sistemas de los niños en riesgo. En este contexto, es muy importante que en los sitios contaminados se controle la exposición desde las fuentes, dado que se ha demostrado que no es suficiente la eliminación de la exposición doméstica (limpieza de espacios infantiles en escuelas y hogares). ${ }^{56}$

Bajo el escenario planteado en el presente trabajo, se demuestra la urgente necesidad de que la exposición a plomo sea tomada en cuenta como un problema nacional de salud pública, cuya atención debe darse en al menos tres ámbitos: el ambiental, para el control de las fuentes de exposición; el sanitario, para la vigilancia de los niveles sanguíneos de plomo en los niños de alto riesgo (por ejemplo, aquellos que vivan en áreas potencialmente contaminadas), ${ }^{28}$ y el educativo, para la atención de niños con problemas neuroconductuales y neurocognitivos, por supuesto considerando que el plomo no es el único neurotóxico y que estos efectos neurológicos en los niños pueden deberse además a otros factores como la desnutrición, por ejemplo. Asimismo, sería de utilidad que en áreas impactadas por plomo el personal de salud estuviera atento a evaluar aquellos efectos asociados con la exposición a este metal (daño cardiovascular, alteracio- 
nes renales, entre otras). ${ }^{57}$ Un primer paso en este sentido lo están dando el Fondo Nacional para el Fomento de las Artesanías y el Instituto Blacksmith en la atención de comunidades alfareras, hecho que resalta al tomar en cuenta que, por ejemplo, la intervención en La Trinidad tiene un carácter de urgente. ${ }^{58}$ Ahora bien, en todos estos ámbitos tendría que haber propuestas de comunicación de riesgo con la capacidad de incorporar programas de autoprotección para disminuir la exposición a plomo; al respecto, ya se cuenta con experiencias en México ${ }^{59}$ y en otros países como Estados Unidos. ${ }^{60-61}$

En cuanto a la investigación, deben apoyarse los trabajos en al menos tres áreas: a) los estudios longitudinales que son útiles para establecer valores límites del plomo en sangre para diversos efectos en la salud y que también cuentan con potencial para medir la interacción del plomo con otros factores de vulnerabilidad (nutrición, características de la madre $)^{62} \mathrm{o}$ con otros tóxicos (por ejemplo, arsénico, flúor, hidrocarburos aromáticos policíclicos, manganeso, compuestos orgánicos persistentes, entre otros) ${ }^{63-65}$ b) evaluación de la capacidad epigenética del plomo para definir los efectos del metal en la etapa adulta como consecuencia de la exposición temprana (por ejemplo, en el período fetal), sobre todo ahora que existen algunos informes que indican una asociación entre enfermedades neurodegenerativas y exposiciones tempranas a plomo; ${ }^{66-67}$ y c) es urgente establecer nuevas normativas de presencia de plomo en el ambiente (aire, agua, suelo, polvo doméstico, polvo urbano, alimentos), que consideren los últimos datos científicos sobre la toxicidad de este elemento, en especial sobre la población infantil. De nada servirá saber que el plomo es tóxico a dosis muy bajas si no se corrigen las normativas que regulan su presencia en el ambiente. Al respecto, urge definir una normativa para presencia de plomo en interiores y exteriores, pues como lo demuestra el presente trabajo, el polvo es una importante ruta de exposición infantil (los valores en esta matriz ambiental correlacionaron con los niveles de plomo en sangre en los niños).

Las consecuencias de no prestar atención a la exposición al plomo son grandes y no solamente en términos de salud para los niños expuestos. El costo económico también ha sido medido. En los Estados Unidos el costo actual de la intoxicación por plomo es de 43000 millones de dólares ${ }^{68} \mathrm{y}$, además, un reciente análisis de costo-beneficio realizado en dicho país encontró que por cada dólar invertido en reducir los riesgos por plomo hay un beneficio de 17 a 220 dólares. ${ }^{69}$ Esta relación es mejor que la obtenida por la aplicación de vacunas que por largo tiempo ha sido descrita como la intervención con el mejor costo-beneficio de la salud pública. ${ }^{69}$
Eliminar el plomo es entonces un asunto de salud pública que merece mantenerse en primer lugar de la atención sanitaria. En un mundo de tópicos ambientales más sofisticados como los cambios climáticos, los tóxicos nanotecnológicos y las basuras electrónicas, el plomo, ahora como antes, se mantiene como un fuerte adversario de la salud infantil y perderlo de vista implica perder de vista el futuro de nuestra nación. Así de simple, así de grave.

\section{Conclusiones}

Los sitios estudiados en el presente trabajo son un ejemplo de las múltiples fuentes de exposición a plomo en México; por consiguiente, se requiere de un programa nacional de salud pública dirigido a reducir la exposición a este metal en poblaciones vulnerables como los niños. Especial énfasis habrá de tenerse en el control desde las fuentes al considerar que un medio de transporte de alto riesgo es el polvo.

\section{Agradecimientos}

Este trabajo fue apoyado por el Consejo Nacional de Ciencia y Tecnología (Fondo Sectorial Salud-2005-C0113841).

Declaración de conflicto de intereses: Los autores declararon no tener conflicto de intereses.

\section{Referencias}

I. World Health Organization. Childhood Lead Poisining. World Health Organization. Geneva: WHO, 2010.

2. International Programme on Chemical Safety. Principles for Evaluating Health Risks in Children Associated with Exposure to Chemicals. Environmental Health Criteria. International Program of Chemical Safety. Geneva: IPCS, 2006.

3. Agency for Toxic Substances and Disease Registry. Toxicological Profile for Lead. Atlanta Georgia: ATSDR, 2007.

4. Fels LM, Wünsch M, Baranowski J, Norska-Borówka I, Price RG, Taylor SA, et al. Adverse effects of chronic low level lead exposure on kidney function--a risk group study in children. Nephrology Dialysis Transplantation 1998; 13: 2248-2256.

5. Gump BB, Stewart P, Reihman J, Lonky E, Darvill T, Matthews KA, et al. Prenatal and early childhood blood lead levels and cardiovascular functioning in $91 / 2$ year old children. Neurotoxicology and Teratology 2005;27: 655-665.

6. Canfield RL, Henderson CR, Cory-Slechta DA, Cox C, Jusko TA, Lanphear BP. Intellectual Impairment in Children with Blood Lead Concentrations below $10 \mu g$ per Deciliter. New England Journal of Medicine 2003;348: I5I7-I526.

7. Lanphear BP, Dietrich K, Auinger P, Cox C. Cognitive deficits associated with blood lead concentrations $<10$ microg/dL in US children and adolescents. Public Health Rep 2000; I 5: 521-529. 
8. Lanphear BP, Hornung R, Khoury J, Yolton K, Baghurst P, Bellinger DC, et al. Low-Level Environmental Lead Exposure and Children's Intellectual Function: An International Pooled Analysis. Environ Health Perspect 2005; I 3 .

9. Téllez-Rojo MM, Bellinger DC, Arroyo-Quiroz C, Lamadrid-Figueroa H, Mercado-García A, Schnaas-Arrieta L, et al. Longitudinal Associations Between Blood Lead Concentrations Lower Than $10 \mu \mathrm{g} / \mathrm{dL}$ and Neurobehavioral Development in Environmentally Exposed Children in Mexico City. Pediatrics 2006; I 18: e323-e330.

10. IPCS. Global Alliance to Eliminate Lead in Paints20II. [Consultado: ] Disponible en: http://www.who.int/ipcs/features/pb_alliance/en/.

II. JECFA. Seventy-third meeting summary and conclusions Joint FAO/WHO Expert Committee on Food Additives. Switzerland 2010. [Consultado: 201 I septiembre] Disponible en: http://www.who.int/ foodsafety/publications/chem/summary73.pdf.

12. Environmental Protection Agency. National Ambient Air Quality Standards for Lead. Federal Register. Washignton: EPA, 2008.

13. Flores J, Albert LA. Environmental lead in Mexico, 1990-2002. Rev Environ Contam Toxicol 2004;181: 37-109.

14. Acosta-Saavedra LC, Moreno ME, Rodriguez-Kessler T, Luna A, Arias-Salvatierra D, Gomez R, et al. Environmental exposure to lead and mercury in Mexican children: a real health problem. Toxicol Mech Methods 20II;2I: 656-666.

15. Afeiche M, Peterson KE, Sánchez BN, Cantonwine D, LamadridFigueroa $\mathrm{H}$, Schnaas L, et al. Prenatal Lead Exposure and Weight of 0 - to 5-Year-Old Children in Mexico City. Environ Health Perspect 20I I; 19.

16. Kordas K, Casavantes KM, Mendoza C, Lopez P, Ronquillo D, Rosado JL, et al. The Association Between Lead and Micronutrient Status, and Children's Sleep, Classroom Behavior, and Activity. Arch Environ Occup Health 2007;62: 105-II2.

17. López-Carrillo L, Torres-Sánchez L, Garrido F, Papaqui-Hernández J, Palazuelos-Rendón E, López-Cervantes M. Prevalence and determinants of lead intoxication in Mexican children of low socioeconomic status. Environ Health Perspect 1996; 104.

18. Romieu I, Carreon T, Lopez L, Palazuelos E, Rios C, Manuel Y, et al. Environmental urban lead exposure and blood lead levels in children of Mexico City. Envir Environ Health Perspect 1995; 103: 1036- 1040.

19. Schnaas L, Rothenberg SJ, Flores M-F, Martinez S, Hernandez C, Osorio $\mathrm{E}$, et al. Reduced Intellectual Development in Children with Prenatal Lead Exposure. Environ Health Perspect 2005; I 14.

20. Zhang A, Hu H, Sánchez BN, Ettinger AS, Park SK, Cantonwine D, et al. Association between Prenatal Lead Exposure and Blood Pressure in Female Offspring. Environ Health Perspect 20I2; I 20(3):445-450.

21. Trejo-Acevedo A, Díaz-Barriga F, Carrizales L, Domínguez G, Costilla $\mathrm{R}$, lze-Lema I, et al. Exposure assessment of persistent organic pollutants and metals in Mexican children. Chemosphere 2009;74: 974-980.

22. Rubio-Andrade M, Valdes-Perezgasga F, Alonso J, Rosado J, Cebrian M, Garcia-Vargas G. Follow-up study on lead exposure in children living in a smelter community in northern Mexico. Environmental Health 201 I; 10: 66.

23. Soto-Jiménez MF, Flegal AR. Childhood lead poisoning from the smelter in Torreón, México. Environmental Research 20 I I; I I : 590-596. 24. Ornelas-Hicks M, Sanín-Aguirre LH, Díaz-Barriga F, Reza-López S, Romieu I. Evaluación de riesgo de intoxicación por plomo en la zona urbana aledaña a una fundidora en Chihuahua, México. Tecnociencia 2006; I: 26-35.

25. Carrizales L, Razo I, Téllez-Hernández JI, Torres-Nerio R, Torres $A$, Batres LE, et al. Exposure to arsenic and lead of children living near a copper-smelter in San Luis Potosi, Mexico: Importance of soil contamination for exposure of children. Environmental Research 2006; 10 I: I-I0. 26. Environmental Protection Agency. Method 305I. Microwave assisted acid digestion of sediments, sludges, soils and oils. Washington: EPA, 2007 27. DOF. NOM-I 47 SEMARNAT/SSAI-2004. Diario Oficial de la Federación. México: Secretaría de Medio Ambiente y Recursos Naturales, 2007.
28. Romieu I. [Use of blood lead data to evaluate and prevent childhood lead poisoning in Latin America]. Salud Publica Mex 2003;45 Suppl 2: S244-25I.

29. Subramanian KS. Determination of lead in blood by graphite furnace atomic absorption spectrometry - A critique. Science of The Total Environment 1989;89: 237-250.

30. Hubaux A, Vos G. Decision and detection limits for calibration curves. Analytical Chemistry 1970;42: 849-855.

31. Miller JC, Miller JN. Estadística y quimiometría para química analítica. $3^{\mathrm{a}}$ ed ed. España: Pretice Hall, 2000: 278

32. DOF. NOM-I99-SSAI-2000, salud ambiental. Niveles de plomo en sangre y acciones como criterios para proteger la salud de la población expuesta no ocupacionalmente. México: Secretaría de Salud, 2000. 33. González-Valdez E, González-Reyes E, Bedolla-Cedeño C, ArrolloOrdaz EL, Manzanares-Acuña E. Niveles de plomo en sangre y factores de riesgo por envenenamiento de plomo en niños mexicanos. Rev Fac Ing Univ Antioquia 2008;43: I I4-II9.

34. Astete J, Cáceres W, Gastañaga MC, Lucero M, Sabastizagal I, Oblitas $\mathrm{T}$, et al. Intoxicación por plomo y otros problemas de salud en niños de poblaciones aledañas a relaves mineros. Rev Perú Med Exp Salud Pública 2009;26: 15-19.

35. Ávalos-Lozano JA. Formación del paisaje minero en el altiplano potosino: siglos XVIII y XIX San Luis Potosí: Universidad Autónoma de San Luís Potosí, 2009.

36. Isla-jiménez C. El real de Tlalpujahua: aspectos de la minería novohispana. México: Instituto Nacional de Antropología e Historia, 2008.

37. Cámara Minera de México. Informe anual 20II. México: Cámara Minera de México, 2011.

38. Albalak R, McElroy RH, Noonan G, Buchanan S, Jones RL, Flanders $W D$, et al. Blood lead levels and risk factors for lead poisoning among children in a Mexican smelting community. Arch Environ Health 2003;58: 172-183.

39. Diaz-Barriga F, Batres L, Calderon J, Lugo A, Galvao L, Lara I, et al. The El Paso smelter 20 years later: residual impact on Mexican children. Environ Res 1997;74: II-16.

40. Garcia-Vargas GG, Rubio-Andrade M, Del Razo LM, Borja-Aburto $\mathrm{V}$, Vera- Aguilar E, Cebrian ME. Lead exposure in children living in a smelter community in region Lagunera, Mexico. J Toxicol Environ Health A 2001;62: 417-429.

4I. Landrigan PJ, Baker EL. Exposure of children in heavy metals from smelters: epidemiology and toxic consequences. Environ Res 1981;25: 204-224. 42. Roberts TM, Hutchinson TC, Paciga J, Chattopadhyay A, Jervis RE, VanLoon J, et al. Lead contamination around secondary smelters: estimation of dispersal and accumulation by humans. Science 1974;186: II20-1I23.

43. Wilson D, Esterman A, Lewis M, Roder D, Calder I. Children's blood lead levels in the lead smelting town of Port Pirie, South Australia. Arch Environ Health 1986;4I: 245-250.

44. INE. Lo que usted debe saber sobre el plomo 2007. [Consultado: 201 I marzo]. Disponible en: http://www2.ine.gob.mx/publicaciones/ folletos/98/98.html.

45. Occupational-Knowledge-International-and-Fronteras-Comunes. Exporting hazards. U.S. shipments of used lead batteries to Mexico take advantage of lax environmental and worker health regulations. San Francisco: Occupational Knowledge International, 201I.

46. Rosenthal E. Lead from old US batteries sent to Mexico raises risks. New York: The New York Times, $201 \mathrm{I}$.

47. Wu Y, Huang Q, Zhou X, Hu G, Wang Z, Li H, et al. [Study on the effects of lead from small industry of battery recycling on environment and children's health]. Zhonghua Liu Xing Bing Xue Za Zhi 2002;23: |67-171.

48. Trejo $O$. Problemática ambiental de la actividad de fabricación artesanal de ladrillos. Congreso de ladrillo artesanal. 2010 oct 6-8; Guanajuato, México. 
49. Artesanías Mexicanas. Alfareros en riesgo de intoxicación por plomo: Notimex 20II. [Consultado en 20II, septiembre 2I]. Disponible en http://www.artesaniasmexicanas.com/noticias/alfareros-en-riesgo-deintoxicación-por-plomo.htm.

50. Hernandez-Serrato MI, Mendoza-Alvarado LR, Rojas-Martinez R, Gonzalez-Garza C, Hulme JM, Olaiz-Fernandez G. Factors associated with lead exposure in Oaxaca, Mexico. J Expo Anal Environ Epidemiol 2003; I3: 34I-347.

5I. Molina Ballesteros G, Zúñiga Charles MA, Cárdenas Ortega A, Solís Cámara PR, Solís Cámara PV. Concentracion de plomo en sangre de niños de familias alfareras. Bol Of Sanit Panam 1982;92: 33-40.

52. SEMARNAT. Registro de Emisiones y Transferencias de Contaminantes. Secretaria del Medio Ambiente y Recursos Naturales. 2009. [Consultado: 201 I marzo]. Disponible en: http://app I.semarnat.gob.mx/ retc/retc09pre/retc09/index.php.

53. SSA. Programa de Acción: Salud Ambiental. Secretaría de Salud. México, 2002. [Consultado: 201 I, agosto]. Disponible en: http://www.salud. gob.mx/unidades/cdi/documentos/DOCSAL7I03.pdf

54. SEMARNAT. Sitios contaminados registrados como pasivos ambientales. Subsecretaría de Gestión para la Protección Ambiental DGdGIdMyAR. Secretaría de Medio Ambiente y Recursos Naturales. 2010. [Consultado: 20I I, agosto]. Disponible en: http://aplicaciones.semarnat.gob.mx/estadisticas/compendio2010/archivos/0I_sitiosc/d3_Sitios03_0I.pdf.

55. Tong S, von Schirnding YE, Prapamontol T. Environmental lead exposure: a public health problem of global dimensions. Bull World Health Organ 2000;78: 1068-1077.

56. Boreland F, Lesjak M, Lyle D. Evaluation of home lead remediation in an Australian mining community. Sci Total Environ 2009;408: 202-208.

57. Leal ME, Gonzalez D, Garcia SI, Perez-Maldonado IN, Rico E, Cossio $P$, et al. [Community clinical toxicology]. Cien Saude Colet 201 I;16: 3503-3508.

58. FONART. Informe 2010 uso de Plomo en la Alfarería en México. Fondo Nacional para el Fomento de las Artesanías. 2010. [Consultado: marzo $20 \mathrm{II}$ ]. Disponible en: www.alfareria.org.

59. Cubillas-Tejeda AC, Torres-Nerio R, Diaz-Barriga Martinez F, Carrizales-Yanez L, Coronado-Salas C, Nieto Caraveo LM, et al. [De- signing and application of a Risk Communication Program for children environmental health on a lead and arsenic contaminated location]. Cien Saude Colet 20II;16: 4II5-4I26.

60. ATSDR. ToxFAQs, Plomo (Lead). Agency for Toxic Substances \& Disease Registry Plomo (Lead). 2007. [Consultado: enero 2012]. Disponible en: http://www.atsdr.cdc.gov/es/toxfaqs/es_tfacts /3.html.

6I. EPA. Lead in paint, dust and soil. Environmental Protection Agency. 2010. [Consultado: enero 2012]. Disponible en: http://www.epa.gov/lead/ pubs/leadinfoesp.htm.

62. Surkan PJ, Schnaas L, Wright RJ, Tellez-Rojo MM, Lamadrid-Figueroa $\mathrm{H}, \mathrm{Hu} \mathrm{H}$, et al. Maternal self-esteem, exposure to lead, and child neurodevelopment. Neurotoxicology 2008;29: 278-285.

63. Bellinger DC. Children's cognitive health: the influence of environmental chemical exposures. Altern Ther Health Med 2007;। 3: SI40-I 44. 64. Cory-Slechta DA. Studying Toxicants as Single Chemicals: Does this Strategy Adequately Identify Neurotoxic Risk? Neurotoxicology 2005;26: 49I-5I0.

65. Henn BC, Schnaas L, Ettinger AS, Schwartz J, Lamadrid-Figueroa $H$, Hernandez-Avila $M$, et al. Associations of early childhood manganese and lead coexposure with neurodevelopment. Environ Health Perspect 2012; |20: |26-|3|.

66. Landrigan PJ, Sonawane B, Butler RN, Trasande L, Callan R, Droller D. Early Environmental Origins of Neurodegenerative Disease in Later Life. Environ Health Perspect 2005; I I3.

67. Weiss B. Lead, Manganese, and Methylmercury as Risk Factors for Neurobehavioral Impairment in Advanced Age. Int J Alzheimers Dis 2011. doi: $10.4061 / 2011 / 607543$.

68. Landrigan PJ, Schechter CB, Lipton JM, Fahs MC, Schwartz J. Environmental pollutants and disease in American children: estimates of morbidity, mortality, and costs for lead poisoning, asthma, cancer, and developmental disabilities. Environ Health Perspect 2002; I 10: 72I-728. 69. Gould E. Childhood Lead Poisoning: Conservative Estimates of the Social and Economic Benefits of Lead Hazard Control. Environ Health Perspect 2009; II7. 